\title{
Reconstrucción nasal con colgajos locales: Manejo según unidades estéticas.
}

*Correspondencia:

drajaramillovera@gmail.com

Teléfono [593] 0980767577

Conflicto de intereses: Los autores declaran no tener conflictos de intereses.

Fondos: Ver la página 204

Recibido: 7 Junio 2015

Aceptado: 11 Diciembre 2016

Publicado: 30 Diciembre 2017

Membrete bibliográfico: Jaramillo $P$, Loor G, Wong $P$, Arévalo $\mathrm{A}$, Almeida $\mathrm{V}$, Chiquito $\mathrm{C}$. Reconstrucción nasal con colgajos locales: Manejo según unidades estéticas. Rev. Oncol. Ecu 2017;27(3):195-205.

DOI: https://doi.org/10.33821/196

Copyright Jaramillo, et al. Este artículo es distribuido bajo los términos de Creative Commons Attribution License, el cual permite el uso y redistribución citando la fuente y al autor original.

\section{Nasal reconstruction Management according to aesthetic units.}

\author{
Priscilla Jaramillo Vera1* id , Gabriel Loor Brocell2, Pamela Wong \\ Lucio², Amy Arévalo Mora $^{3}$, Vanesa Almeida Sacoto $^{3}$, Camila Chiquito \\ Freile ${ }^{3}$.
}

1. Servicio de Partes Blandas del Instituto Oncológico Nacional "Dr. Juan Tanca Marengo" Solca, Guayaquil-Ecuador.

2. Postgrado de Cirugía Plástica Reconstructiva y Estética de la Universidad de Especialidades Espíritu Santo, Guayaquil-Ecuador.

3. Servicio de cirugía del Instituto Oncológico Nacional "Dr. Juan Tanca Marengo" Solca, Guayaquil-Ecuador.

\section{Resumen}

Introducción: Las lesiones neoplásicas de la nariz necesitan una resolución con resultado estético satisfactorio. El objetivo del presente estudio es realizar una descripción del lugar en donde se ubicaron las lesiones y los tipos de colgajos más utilizados.

Métodos: El presente estudio descriptivo fue realizado en pacientes intervenidos en el 2013 por reconstrucción nasal secundaria exéresis tumoral en Solca-Guayaquil. Se describe la localización de la lesión y la técnica. La muestra fue incidental. Para la descripción se dividió la nariz en 3 zonas: proximal (Z1), media (Z2) y distal (Z3). Las lesiones con >1 zona fueron "defectos complejos". Las $\mathrm{Z1}$ y Z2 fueron divididas en subunidades (SU) central y lateral. La Z3 fue dividida en SU: alar, domoalar, de domo, central, columelar y de base nasal. Los defectos con >1 SU fueron combinados.

Resultados: Ingresaron al estudio 96 casos, 60 mujeres. 14 defectos (14.6 \%) en Z1, 16 defectos (16.7\%) en Z2, 50 casos (52\%) en Z3 y 16 defectos complejos (16.7\%). El colgajo frontal (CF) 21 casos (21.88 \%), el colgajo avance $V-Y(A V-Y) 19$ casos (19.79\%) y el colgajo nasolabial (CNL) 15 casos (15.63\%). En Z1, la SU- central se utilizaron los colgajos glabelar (CG), AV-Y y colgajo en Mitra (CM); en la SU- lateral se utilizaron el CG y AV-Y con más frecuencia. En la Z2, la SU- central se utilizaron los $\mathrm{CM}$ y el cierre directo (CD); en la SU- lateral se utilizaron el CD, CM, AV-Y y CNL. En la Z3 se utilizaron $\mathrm{CNL}, \mathrm{AV}-\mathrm{Y}$ y colgajos bilobulados. El colgajo frontal fue usado en los defectos complejos y combinados.

Conclusión: La SU-Alar y la SU del Surco Domal Alar de la Z3, fueron las áreas más prevalentes. Las técnicas quirúrgicas más utilizadas fueron, el colgajo frontal, el AV-Y y el CNL. 
Palabras Claves: CIRUGÍA PLÁSTICA, COLGAJOS QUIRÚRGICOS, RECONSTRUCCIÓN, PROCEDIMIENTOS QUIRÚRGICOS RECONSTRUCTIVOS, NARIZ DEFORMIDADES ADQUIRIDAS NASALES.

DOI: $10.33821 / 196$

\section{Abstract}

Introduction: The neoplastic lesions of the nose need a resolution with a satisfactory aesthetic result. The aim of the present study is to make a description of the place where the injuries were located and the types of flaps most used.

Methods: The present descriptive study was performed in patients who underwent surgery in 2013 for secondary nasal reconstruction of tumor exeresis in Solca-Guayaquil. The location of the lesion and the technique are described. The sample was incidental. For the description, the nose was divided into 3 zones: proximal (Z1), middle (Z2) and distal (Z3). Injuries with> 1 zone were "complex defects". The $\mathrm{Z} 1$ and $\mathrm{Z} 2$ were divided into central and lateral subunits (SU). The Z3 was divided into SU: alar, domo-alar, dome, central, columelar and nasal base. Defects with> $1 \mathrm{SU}$ were combined.

Results: 96 cases, 60 women, entered the study. 14 defects (14.6\%) in Z1, 16 defects (16.7\%) in Z2, 50 cases (52\%) in Z3 and 16 complex defects (16.7\%). The frontal flap (FF) 21 cases (21.88\%), the flap advance $\mathrm{V}-\mathrm{Y}$ (AV-Y) 19 cases (19.79\%) and the nasolabial flap (NLF) 15 cases (15.63\%). In Z1, the SU-central glabellar flap (GF), AV-Y and Mitra flap (MF) were used; in the SU-lateral the GF and AV-Y were used more frequently. In the Z2, the central SU were used the MF and the direct closure (DC); in the SU-lateral DC, MF, AV-Y and NLF were used. In Z3, NLF, AV-Y and bi-lobed flaps were used. The frontal flap was used in complex and combined defects.

Conclusion: The SU-Alar and the SU of the Domal Alar Groove of the Z3, were the most prevalent areas. The most used surgical techniques were, the frontal flap, the AV-Y and the NLF.

Keywords: PLASTIC SURGERY, SURGICAL FLAPS, RECONSTRUCTION, RECONSTRUCTIVE SURGICAL PROCEDURES, NOSE ACQUIRED NASAL DEFORMITIES.

DOI: $10.33821 / 196$

\section{Introducción}

La nariz es la proyección más notoria de la cara, por lo que realizar escisión de pequeños defectos puede significar una dificultad técnica para dejar la menor lesión estética posible. Cualquier distorsión de la apariencia nasal es un problema estético para el paciente y debe ser evitada. Todo tipo de cirugía produce una cicatriz, pero puede ser bien tolerada si ésta se encuentra disimulada en los accidentes nasales naturales. La reconstrucción estética de defectos nasales puede ser muy compleja específicamente luego de escisión de neoplasias. Existe un gran número de técnicas quirúrgicas que han sido descritas para la reconstrucción nasal, resultando difícil la elección de éstas para cualquier defecto en particular teniendo como objetivo el que pase desapercibida su reconstrucción en cuanto a resultado estético [1]. 
Estos métodos de reconstrucción pueden ser a menudo intercambiables, pero ciertos colgajos funcionan mejor en áreas específicas de la nariz, por ejemplo los defectos horizontales son mejor reconstruidos con colgajos que se mueven hacia abajo como el colgajo glabelar, o en "mitra"; y en los defectos verticales se obtienen mejores resultados con colgajos $\mathrm{V}-\mathrm{Y}$ y nasolabiales. El objetivo del presente estudio es realizar una descripción del lugar en donde se ubicaron las lesiones y los tipos de colgajos más frecuentes utilizados en un grupo de pacientes con lesiones post exéresis tumorales neoplásicas en la pirámide nasal.

\section{Materiales y Métodos}

El presente estudio es descriptivo, retrospectivo, el cual fue realizado en pacientes intervenidos en el año 2013 por reconstrucción nasal secundaria exéresis tumoral en el servicio de partes blandas del Instituto Oncológico Nacional "Dr. Juan Tanca Marengo" Solca-Guayaquil. Se describe la localización y técnicas usadas para reconstruirlos. La muestra fue no probabilística, de tipo incidental del período de estudio. El estudio contó con la aprobación del departamento de docencia de la institución. Se recurrió al archivo fotográfico del Servicio de Partes Blandas para documentar las técnicas de reconstrucción nasal. Se excluyeron los pacientes con patologías vasculares, tabaquismo y aquellos a los cuales se sometieron a reconstrucción nasal en otro centro hospitalario.

Se mapeó los defectos de acuerdo a la localización de los mismos en la nariz. Para el propósito de los análisis arbitrariamente se dividió la nariz en 3 zonas: proximal, media y distal (Figura 1). Las lesiones que incluyen más de 2 zonas fueron denominados "defectos complejos" (Figura 1). Las zonas proximal y media fueron divididos en subunidades central y lateral. La zona distal fue dividida en subunidades: alar, domo-alar, de domo, central, columelar y de base nasal. Los defectos que tomaron más de una subunidad fueron categorizados como defectos combinados.

Figura 1. División de las lesiones en la pirámide nasal

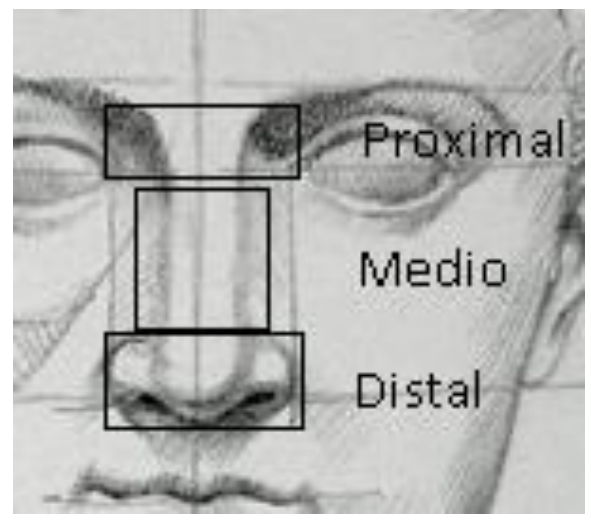

Se describe la frecuencia en cada zona anatómica. Los diagramas del diseño del colgajo para cada opción reconstructiva fueron descritos como se han reportado en estudios 
previos: glabelar [1], colgajo de avance $\mathrm{V}-\mathrm{Y}$ [2], bilobulado [3], colgajos nasolabiales [4] y colgajo en Mitra [5].

\section{Resultados}

Se incluyeron en el estudio 15 pacientes, 10 mujeres (66.7\%), con edad media de 50 años (35 - 65 años). La localización de cáncer más frecuente fue colon-rectal $33 \%$, seguido por otros tipos de cáncer 6 \% (plasmocitoma, mieloma múltiple, leiomiosarcoma). El Índice de Karnosky al inicio del estudio en la totalidad de los pacientes dio una media de 78 \% (60 90) (Tabla 1). En esta serie todos los casos fueron reportados el estudio patológico transquirúrgico con márgenes libres de tumor.

\section{Tabla 1. Frecuencia de técnicas quirúrgicas}

\begin{tabular}{|l|c|c|}
\hline & $\mathrm{n}=\mathbf{9 6}$ & Porcentaje \\
\hline Colgajo frontal & 21 & 21.88 \\
\hline Avance V-Y & 19 & 19.79 \\
\hline Colgajo nasolabial & 15 & 15.63 \\
\hline Colgajo en mitra & 11 & 11.46 \\
\hline Colgajo bilobulado & 11 & 11.46 \\
\hline Cierre directo & 8 & 8.33 \\
\hline Colgajo de glabela & 4 & 4.17 \\
\hline Avance de mejilla & 2 & 2.08 \\
\hline Injerto total & 2 & 2.08 \\
\hline Colgajo local & 2 & 2.08 \\
\hline Injerto piel parcial & 1 & 1.04 \\
\hline
\end{tabular}

Defectos en la zona proximal: De los 14 defectos del tercio proximal, la mayor prevalencia fue en la subunidad lateral 7 casos (50 \%), 4 fueron defectos combinados (28.6 \%). La mayoría de lesiones fueron resueltos por colgajos frontales, colgajos glabelares y avance V-Y (Tabla 2).

Tabla 2. Defectos de la zona proximal

\begin{tabular}{|l|c|c|c|c|}
\hline & S. Central & S. Lateral & D. Combinados & Total \\
\hline Colgajo frontal & - & - & 4 & 4 \\
\hline Colgajo Glabelar & 1 & 2 & - & 3 \\
\hline Avance V-Y & 1 & 2 & - & 3 \\
\hline Mitra & 1 & 1 & - & 2 \\
\hline Injerto & - & 1 & - & 1 \\
\hline Cierre directo & - & 1 & - & 1 \\
\hline Total & 3 & 7 & 4 & 14 \\
\hline
\end{tabular}

D=Defectos, $S=$ Subunidad. 
Defectos en la zona media: De los 16 defectos en el tercio medio, la mayor prevalencia de lesiones se ubicó en la subunidad lateral 8 casos (50\%). La mayoría de lesiones fueron resueltas con cierre directo y colgajo en mitra (Tabla 3 ).

Tabla 3. Defectos de la zona media

\begin{tabular}{|l|c|c|c|c|}
\hline & S. Central & S. Lateral & Defectos Combinados & Total \\
\hline Cierre directo & 2 & 2 & - & 4 \\
\hline Colgajo en mitra & 2 & 2 & - & 4 \\
\hline Avance V-Y & - & 2 & - & 2 \\
\hline Colgajo nasolabial & - & 2 & - & 2 \\
\hline Colgajo Frontal & - & - & 2 & 2 \\
\hline Colgajo Bilobulado & - & - & 1 & 1 \\
\hline Avance de mejilla & - & - & 1 & 1 \\
\hline Total & 4 & 8 & 4 & 16 \\
\hline
\end{tabular}

Defectos en la zona distal: En el tercio distal se encontraron 50 defectos. Los defectos en la zona distal fueron más prevalentes en la subunidad alar 14 casos $(28 \%)$, del surco domal alar 10 casos $(20 \%)$ y la subunidad central 9 casos (18\%). Las técnica quirúrgicas más frecuentemente usadas en esta zona fueron el Colgajo nasolabial en 12 casos (24\%), el Avance $V-Y$ en 12 casos (24\%) y el colgajo bilobulado en 9 casos (18\%) (Tabla 4).

Figura 2. Defectos del tercio proximal
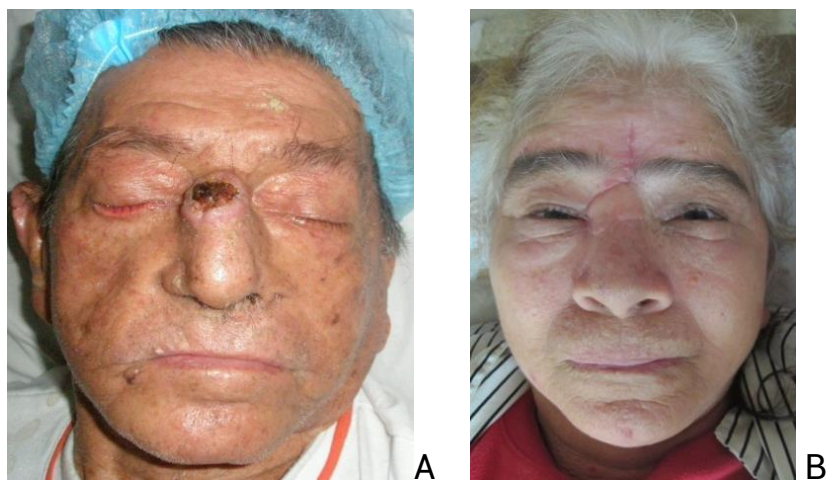

A) Paciente con gran neoplasia que por su tamaño y localización, fue requerido un colgajo frontal para su cobertura. B) Paciente con lesión que por su localización y tamaño menor fue reconstruida con un colgajo glabelar.

Defectos complejos: Se tomó 20 defectos complejos en esta serie. Cuatro de ellos comprometían el tercio medio y proximal y fueron reconstruidos con colgajos frontales, un bilobulado y uno de glabela. Siete envolvían el tercio medio y distal reconstruyéndolos con 2 colgajos frontales, dos $\mathrm{V}-\mathrm{Y}$ de avance, un nasolabial, un avance de mejilla y un colgajo en mitra. Fueron 5 defectos que envolvían las tres zonas, se usó completamente el colgajo frontal (Tabla 5). 
Tabla 4. Defectos en la zona distal

\begin{tabular}{|l|c|c|c|c|c|c|c|c|}
\hline & $\mathbf{1}$ & $\mathbf{2}$ & $\mathbf{3}$ & $\mathbf{4}$ & $\mathbf{5}$ & $\mathbf{6}$ & $\mathbf{7}$ & Total \\
\hline Colgajo nasolabial & 7 & 3 & - & $\mathbf{1}$ & - & - & $\mathbf{1}$ & 12 \\
\hline Avance V-Y & 7 & 2 & 2 & 1 & - & - & - & 12 \\
\hline Colgajo bilobulado & - & 1 & 4 & 4 & - & - & - & 9 \\
\hline Colgajo frontal & - & - & - & - & 6 & - & & 6 \\
\hline Colgajo en mitra & - & 1 & 2 & 1 & - & - & - & 4 \\
\hline Cierre directo & - & 2 & - & 1 & - & - & - & 3 \\
\hline Colgajo local & - & - & - & - & - & 2 & - & 2 \\
\hline Injerto total de piel & - & - & 1 & - & - & - & - & 1 \\
\hline Injerto parcial de piel & - & 1 & - & - & - & - & - & 1 \\
\hline Total & 14 & 10 & 9 & 8 & 6 & 2 & 1 & 50 \\
\hline
\end{tabular}

1: SU Alar. 2: SU Surco Domal Alar. 3: SU Central. 4: SU Domo. 5: Defectos Combinados. 6: SU Columela. 7: SU base nasal.

Figura 3. Defectos del tercio medio.
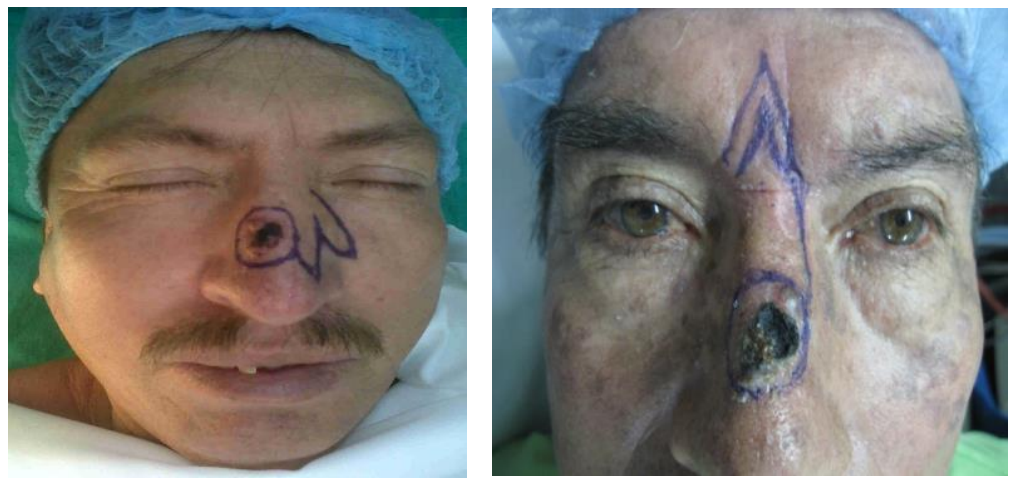

Defectos de las mismas características pero con distintas maneras de cobertura: a la izquierda observamos un colgajo bilobulado y a la derecha un diseño de colgajo mitral.

Tabla 5. Defectos complejos

\begin{tabular}{|l|c|c|c|c|}
\hline & $\begin{array}{l}\text { Zona Proximal } \\
\text { y Media }\end{array}$ & $\begin{array}{l}\text { Zona } \\
\text { media } \\
\text { distal }\end{array}$ & $\begin{array}{l}\text { yona } \\
\text { Proximal- } \\
\text { Media y distal }\end{array}$ & Total \\
\hline Colgajo frontal & 2 & 2 & 5 & 9 \\
\hline Avance V-Y & - & 2 & - & 2 \\
\hline Colgajo bilobulado & 1 & - & - & 1 \\
\hline Colgajo de glabela & 1 & - & - & 1 \\
\hline Colgajo Nasolabial & - & 1 & - & 1 \\
\hline Avance de mejilla & - & 1 & - & 1 \\
\hline Colgajo en mitra & - & 1 & - & 1 \\
\hline Total & 4 & 7 & 5 & 16 \\
\hline
\end{tabular}


Figura 4. Defectos del tercio distal
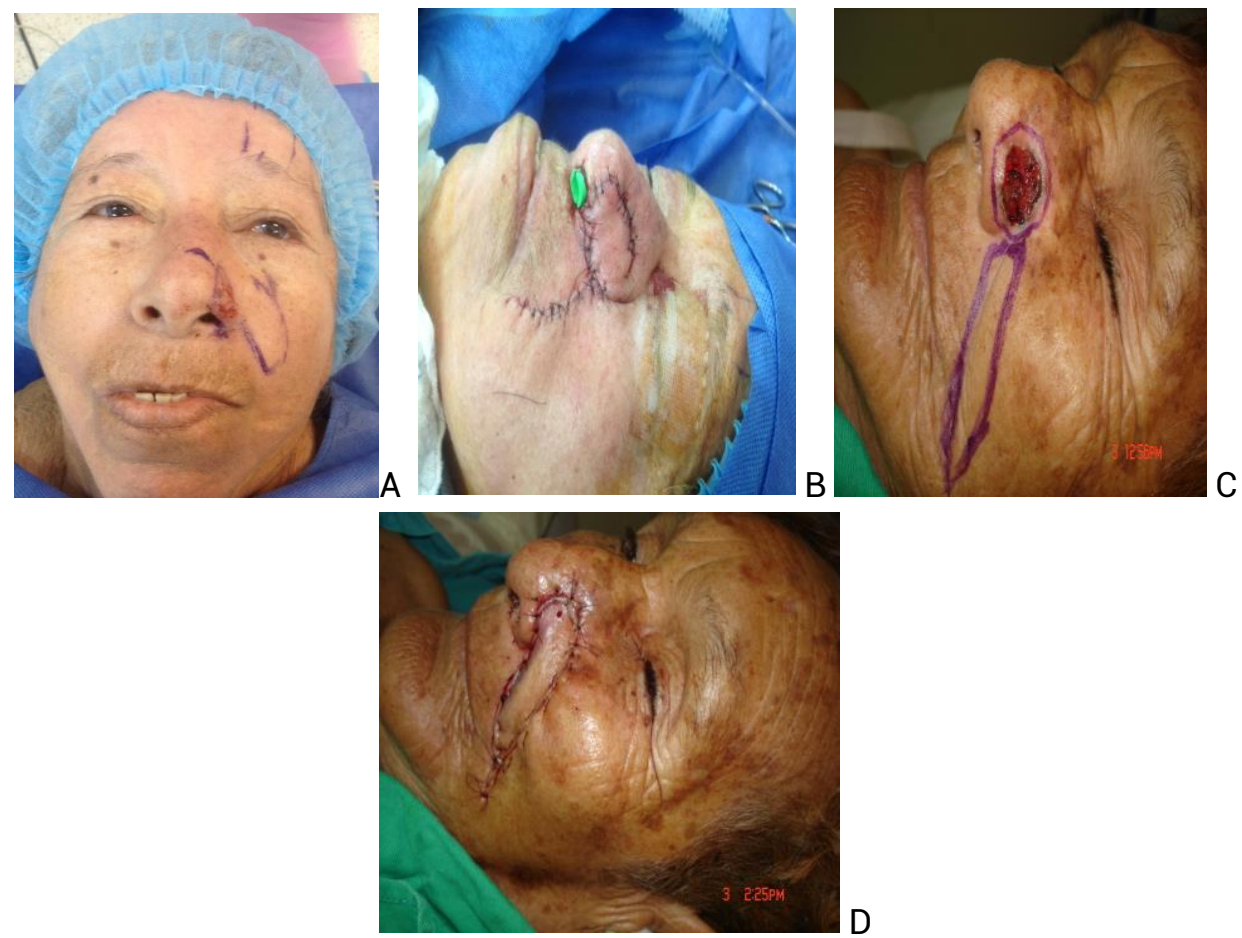

Se observa defecto en ala nasal izquierda de diverso manejo. Ay B) Se realiza colgajo nasogeniano con buen resultado estético. C y D) Se realizó avance $V-Y$. Ambos colgajos tienen un gran nivel de seguridad para el cirujano plástico.

Figura 5. Neoplasia compuesta
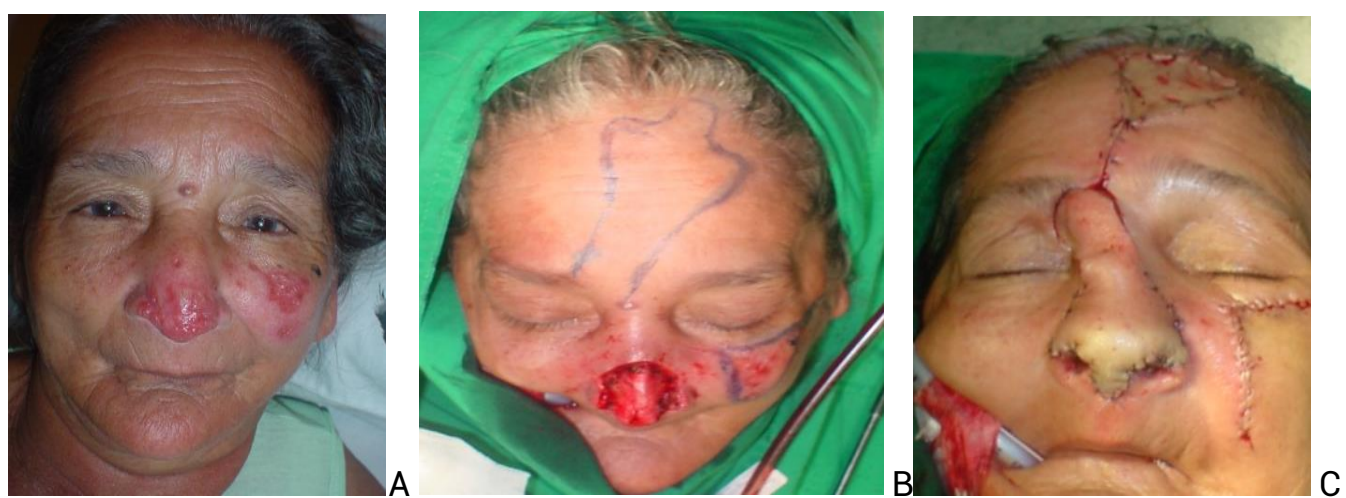

Evolución de paciente que involucra neoplasia compuesta de tercio distal hasta columela, realizada la exéresis de la misma, se procede a realizar colgajo frontal con buen resultado estético. 


\section{Discusión}

En este reporte la población femenina tiene una mayor prevalencia, con una relación 2:1 femenino-masculino. Los defectos en el tercio distal fueron los más prevalentes, esto contrasta con lo publicado previamente en donde se describe que la base proximal de la nariz es el sitio más frecuente que incluye las neoplasias faciales [6, 7]. En esta serie ninguna reconstrucción se realizó sin la confirmación del estudio patológico transquirúrgico de contar con márgenes libres de tumor. Este algoritmo realizado puede ser tomado para cualquier tipo de reconstrucción nasal independientemente de su patología (amputación, trauma, agenesia) y es el algoritmo propuesto por Pribar y colaboradores [6].

Tercio proximal: Cuando se reconstruye defectos de la subunidad central de la zona proximal, un colgajo glabelar o en mitra deberían ser la recomendación. Topográficamente, la geometría del colgajo glabelar es muy similar al colgajo mitral, el primero se recomienda para defectos superiores y el segundo para defectos cerca del tercio medio. En la subunidad lateral, se recomienda los colgajos glabelares y avances $V-Y$. Los defectos con orientación vertical pueden ser cerrados con avance $V-Y$; en cambio si la orientación transversa, un colgajo glabelar o en mitra es la mejor opción. Para los defectos combinados debemos aplicar un colgajo frontal [6].

Tercio Medio: Está reportado que en los defectos centrales del tercio medio, los colgajos en mitra pueden ser usados para cerrar la mayoría de ellos, sin embargo en este reporte la opción de cierre directo a igual que el colgajo en mitra fueron los más prevalentes en la serie. En la subunidad lateral si el defecto es orientado transversalmente, un colgajo en mitra debe ser usado, aunque también puede ser utilizado un avance $\mathrm{V}-\mathrm{Y}$, un colgajo nasolabial o un bilobulado. Escoger entre un avance $V-Y$ y un colgajo nasolabial depende de la preferencia del cirujano. Un colgajo nasolabial va mejor para un defecto lateral y un defecto central en este punto puede ser cubierto con un colgajo $\mathrm{V}-\mathrm{Y}$. Los colgajos frontales pueden ser necesarios en defectos combinados.

Zona distal: Distalmente, colgajos nasolabiales y avances $\mathrm{V}-\mathrm{Y}$ pueden ser realizados para defectos que involucren la región alar o surco domal-alar. Si un colgajo V-Y es seleccionado para el cierre, es importante que el complejo alar no sea irrumpido y que el diseño del colgajo sobre el surco alar y se extienda dentro del área nasolabial, asegurando el suplemento sanguíneo de los perforadores de la arteria angular. Frecuentemente un colgajo V-Y es necesario para reconstruir estos defectos. Para tales defectos, un colgajo nasolabial puede ser usado, pero una división secundario y retoque será requerido; generalmente los colgajos $\mathrm{V}-\mathrm{Y}$ son procedimientos de un solo tiempo.

Centralmente, en el domo y en la subunidad central, un colgajo bilobulado es el colgajo de elección. El área domal es especialmente propensa a distorsión, y se debe rotar lo menos posible la punta en reconstrucciones. A través del tercio distal, los colgajos en mitra continúan siendo la elección cuando el defecto tiene orientación transversa. La columela es difícil de reconstruir, y hemos tomado la iniciativa de si está comprometida, retirar toda la unidad estética y usar un colgajo frontal dando mejor resultado estético. La base nasal 
puede ser reconstruida con colgajo nasolabial perforante en isla. Para defectos combinados del tercio distal la mejor opción continua siendo el colgajo frontal.

Defectos complejos: El colgajo frontal se mantiene como puntal en el tratamiento de defectos que involucran más de una subunidad, especialmente cuando 2 a 3 zonas están involucradas. Cuando un colgajo frontal es requerido, seguiremos los principios expuestos por Burget y Menick, apuntando a reconstruir la subunidad entera para máximo resultado estético [8]. Cuando tenemos defectos pequeños no debemos poner mucha atención al principio de la subunidad porque esto incrementa la demanda reconstructiva necesaria pudiendo acabar en un colgajo frontal innecesario [9].

La satisfacción estética en cuanto a reconstrucción puede ser lograda, proveyendo atención al restaurar el contorno nasal [10. En este artículo se describe la gran cantidad de colgajos para cobertura de región nasal, encontrando un algoritmo basado en la localización, y orientación del defecto; la valoración objetiva del archivo fotográfico. Estos colgajos dieron mejor resultado estético, comparándolos con otros utilizados en la misma región.

\section{Conclusiones}

La subunidad alar y la subunidad del surco domal alar de la zona del tercio distal, fueron las áreas más prevalentes de lesiones en las que se realizó correcciones quirúrgicas. Las técnicas quirúrgicas más utilizadas fueron El colgajo frontal, el colgajo avance $\mathrm{V}-\mathrm{Y}$ y el colgajo nasolabial.

\section{Agradecimientos}

Queremos agradecer el apoyo institucional para la realización del estudio así como los trabajadores y pacientes del Instituto Oncológico Nacional "Dr Juan Tanca Marengo"-Solca Guayaquil.

\section{Información adicional}

\section{Abreviaturas}
AV: Avance
CD: Cierre directo.
CNL: Colgajo nasolabial.
CF: Colgajo frontal.
CM: Colgajo en mitra.
SU: Subunidad. 
Nota del Editor

La Revista Oncología Ecu permanece neutral con respecto a los reclamos jurisdiccionales en mapas publicados y afiliaciones institucionales.
Archivos Adicionales

Ninguno declarado por los autores.

Fondos

Los fondos para la presente investigación fueron propios de los autores del presente artículo.

\section{Disponibilidad de datos y materiales}

Existe la disponibilidad de datos bajo solicitud al autor de correspondencia. No se reportan otros materiales.

\section{Contribuciones de los autores}

AJB y ACR idea de investigación y diseño. ACR, MFP, MBP recolectaron los datos. ACR realizó el análisis estadístico. AJB realizó las cirugías. ACR, MFP y MBP escribieron el artículo. AJB análisis crítico del artículo. Todos los autores leyeron y aprobaron el manuscrito final.

Aprobación de ética y consentimiento para participar

No se aplica a un estudio observacional retrospectivo.

\section{Consentimiento para publicación}

No aplica a un estudio observacional retrospectivo.

\section{Información de los autores}

Priscilla Jaramillo Vera, Cirujano Plástico Tratante del Servicio de Partes Blandas del Instituto Oncológico Nacional "Dr. Juan Tanca Marengo"- Solca, Guayaquil.

(iD) https://orcid.org/0000-0003-1261-9380

Gabriel Loor Brocell, Médico Residente del tercer año de Postgrado de Cirugía Plástica Reconstructiva y Estética de Universidad Espíritu Santo U.E.E.S. 
Pamela Wong Lucio, Médico Residente de Segundo año de Postgrado de Cirugía General de Universidad Espiritu Santo U.E.E.S.

Amy Arévalo Mora, Médico residente del área de Cirugía del Instituto Oncológico Nacional "Dr. Juan Tanca Marengo"- Solca, Guayaquil.

Vanesa Almeida Sacoto, Médica residente del área de Cirugía del Instituto Oncológico Nacional "Dr. Juan Tanca Marengo"- Solca, Guayaquil.

Camila Chiquito Freile, Médica residente del área de Cirugía del Instituto Oncológico Nacional "Dr. Juan Tanca Marengo"- Solca, Guayaquil.

Revisiones por pares

Acceda a la revisión de pares académicos en el siguiente enlace: https://publons.com/review/3148004

Abreviaturas en la referencias

DOI: Digital Object

Identifier

PMID: PubMed Identifier

SU: Short URL

\section{Referencias}

1. Jackson I. Local Flaps in Head and Neck reconstruction. Quality Medical Publishing (2002). RICHMOND, TX, Estados Unidos de America, 13 Edition. ISBN: 9781576261637.

2. Rieger R. A Local Flap for Repair of the Nasal Tip. Plastic and Reconstructive Surgery 1967;40(2):147149. SU: goo.gl/JN5fdP

3. Zitelli J. The bilobed flap for nasal reconstruction. Arch Dermatol. 1989;125(7):957-9. PMID: 2742390

4. Ito O, Yano T, Kawazoe T, Suzuki S. Flexible Curved V-Y Subcutaneous Flap for Facial Skin Defects. Plast Reconstr Surg Glob Open. 2015 Oct 9;3(10):e531. DOI: 10.1097/G0X.0000000000000499.

5. Yoon T, Benito-Ruiz J, García-Díez E, Serra-Renom J. Our algorithm for nasal reconstruction. Journal of Plastic Renconstructive Aesthetic Surgery 2006;59(3):239-247. DOI: 10.1016/j.bjps.2005.09.035

6. Guo L, Pribaz J, Pribaz J. Nasal Reconstruction with Local Flaps: A Simple Algorithm for Management of Small Defects. Plastic and Reconstructive Surgery 2008;122(5):130e-139e. DOl: 10.1097/PRS.0b013e31818823c7

7. Heckmann M, Zogelmeier F, Konz B. Frequency of facial basal cell carcinoma does not correlate with site-specific UV exposure. Arch Dermatol 2002;138(11):1494-7. DOI: 10.1001/archderm.138.11.1494

8. Burget G, Menick F. The subunit principle in nasal reconstruction. Plastic Surgery Journal 1985;76(2):239-247. SU: goo.gl/MRXBYQ

9. Singh DJ, Bartlett SP. Aesthetic considerations in nasal reconstruction and the role of modified nasal subunits. Plast Reconstr Surg. 2003;111(2):639-48. DOI:10.1097/01.PRS.0000041602.85239.93

10. Strom SS, Yamamura Y. Epidemiology of nonmelanoma skin cancer. Clin Plast Surg 1997;24(4):627-36. Review. PMID: 9342506. 